\title{
On-bed Sleep Posture Recognition Based on Body-Earth Mover's Distance
}

\author{
Xiaowei $\mathrm{Xu}^{1,2}$, Feng $\mathrm{Lin}^{2}$, Aosen $\mathrm{Wang}^{2}$, Chen Song ${ }^{2}$, $\mathrm{Yu} \mathrm{Hu}{ }^{1}$, Wenyao $\mathrm{Xu}^{2}$ \\ 1 School of Optical and Electronic Information, HUST, Wuhan, China \\ 2 Department of Computer Science and Engineering, SUNY at Buffalo, New York, USA \\ \{xuxiaowei, bryanyu\}@hust.edu.cn; \{flin28, aosenwan, csong5, wenyaoxu\}@buffalo.edu
}

\begin{abstract}
Sleep quality is highly correlated with many chronic diseases, such as cardiovascular disease and sleep disorders. Sleep posture is a key component in sleep quality assessment. Currently, body pressure analysis has been a popular approach for sleep posture recognition [1]. In this paper, we propose a novel distance metric for sleep posture dissimilarity measure, called Body-Earth Mover's Distance (BEMD). BEMD can effectively recognize the difference of pressure distributions by projecting the pressure distribution to the horizontal and vertical directions. A pilot study is performed with 14 persons for six different postures. The experimental results show that our proposed BEMD can achieve a high accuracy of $90.78 \%$, which outperforms the previous method [1] with an improvement of $9.1 \%$.
\end{abstract}

\section{INTRODUCTION}

As about one third of human life is spent in sleeping to heal the body, sleep quality is of fundamental importance to human health. Sleeping status (such as sleep stage, sleep time, and sleep loss) has a proven relationship with chronic diseases, such as obesity and diabetes [2]. Sleep disorders (such as sleep apnea) is one of the main cause of other serious diseases, e.g., hypertension and bipolar disorder [3].

Sleep posture has been adopted to evaluate the quality of sleep, which is a promising method to provide more insight into sleep. As a crucial indicator for sleep quality, sleep posture has been widely studied for medical diagnoses and sleep disease treatment. For example, Ambrogio et al. [4] reported that the supine (lying on back) should be avoided for patients with chronic respiratory in sufficiency.

Meanwhile, sleep posture also plays an important role for pressure ulcers prevention. Pressure ulcer, i.e., bedsores, is one of the main problems for post-surgical patients and elderly patients. To date, more than 2.5 million people in the United States develop pressure ulcers every year [5]. Pressure ulcer is a serious disease which can trigger other ailments, e.g., bone infection and pyarthroses. The best way to combat pressure ulcers is prevention. The most important care for a person to prevent pressure ulcers is the redistribution of pressures. Thus, nursing homes and hospitals usually set programs in place to change the sleep postures of the patients at risk. However, there is no long-term monitoring to ensure the pressure is distributed in a proper way. Therefore, there is an urgent need for autonomously monitoring sleep postures for medical diagnoses and pressure ulcers prevention.

In the last decade, researchers have proposed several ways to monitor sleep posture autonomously. Video cameras [6] and near-infrared cameras [7] have been used to recognize sleep postures. However, this approach suffers from privacy concerns and image noise due to low visibility at night. Another approach [8] adopted inertial sensors, including accelerometers, gyroscopes, and magnetometers. These sensors need to be attached to the human body, which causes inconvenience for patients during sleep.

Recently, the approach of dispersed pressure sensors embedded in the mattress has become promising. The sensorembedded mattress can record the pressure distribution of human bodies, which forms a pressure image. Compared with other existing solutions (i.e., wearable sensors and cameras), this sensing modality is unobtrusive to users and minimizes the privacy concerns. Liu et al. [1] [9] adopted 32 features of pressure images for classifications of six sleep postures. Ostadabbas et al. [10] made the classification with 13 features of pressure images for three sleep postures. However, these methods for sleep posture recognition are based on local features and individualized pre-training, which requires considerable efforts to apply in a large population.

In this paper, we propose a novel distance metric, BodyEarth Mover's Distance (BEMD) for sleep posture recognition on a pressure sensitive bedsheet. This metric can directly quantify the similarity between sleep postures without specific feature extraction. In our approach, we firstly project the pressure measures to horizontal and vertical directions. Then the similarity between pressure images can be evaluated with the obtained projection histograms. K nearest neighbor $(k \mathrm{NN})$ is used to perform the classification of six sleep postures. We evaluate the proposed approach with real-world data, and our proposed approach outperforms the previous method [1].

\section{BACKGROUND}

We first introduce the Earth Mover's Distance (EMD) for the reader's convenience. Suppose there are $n$ suppliers, $a_{i}$, each with a given amount of goods, $\alpha_{i}$. There are $m$ consumer$\mathrm{s}, b_{j}$ each with a predefined capacity, $\beta_{j}$. The suppliers and consumers with amounts of goods and capacities are represented as $A$ and $B$ for simplification, where $A=\left\{\left(a_{i}, \alpha_{i}\right), 1 \leq\right.$ $i \leq n\}, B=\left\{\left(b_{j}, \beta_{j}\right), 1 \leq j \leq m\right\}$. The total amount of goods in suppliers is equal to the total capacity of consumers. The per-unit good's cost $=\left\{c_{i j}, 1 \leq i \leq n, 1 \leq j \leq m\right\}$ of each supplier-consumer pair is given. The task is to find a flow, $F=\left\{f_{i j}, 1 \leq i \leq n, 1 \leq j \leq m\right\}$, that transports the goods from the suppliers to the consumers with the least cost:

$$
E M D(A, B)=\min \left\{\sum_{i=1}^{n} \sum_{j=1}^{m} c_{i j} f_{i j}\right\},
$$




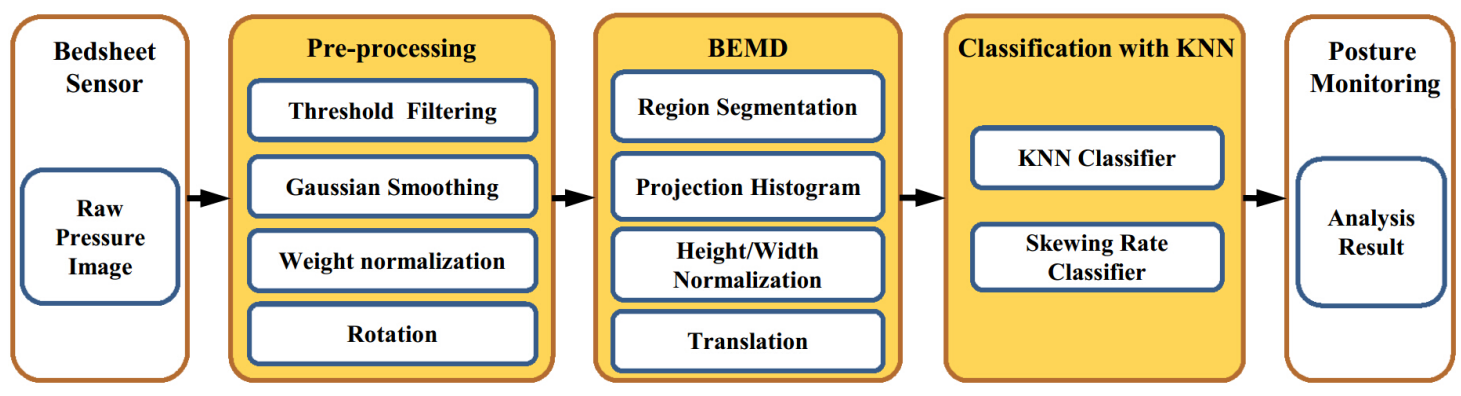

Fig. 2. The sleep posture recognition framework.

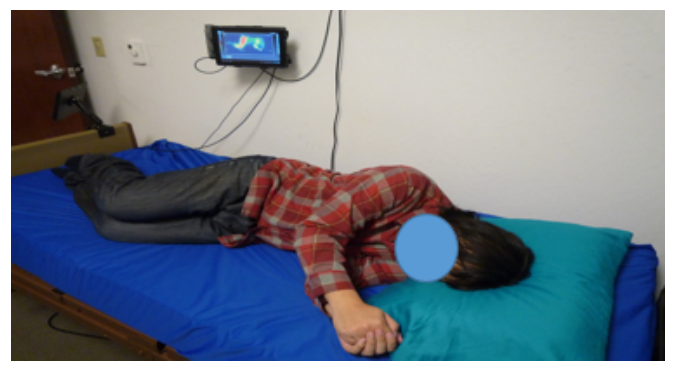

(a)

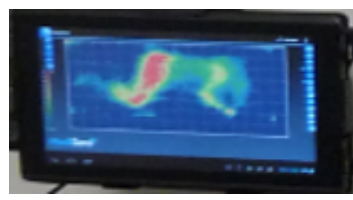

(b)

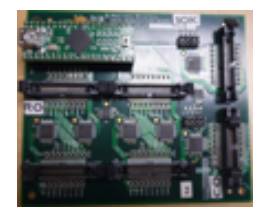

(c)

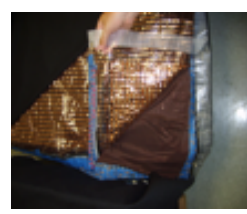

(d)

Fig. 1. (a) The bedsheet system in use and its submodules: (b) tablet, (c) scanning module and (d) E-textile with conductive lines.

with subject to

$$
\begin{gathered}
f_{i j} \geq 0,1 \leq i \leq n, 1 \leq j \leq m, \sum_{i=1}^{n} \alpha_{i}=\sum_{j=1}^{m} \beta_{j}, \\
\sum_{j=1}^{m} f_{i j} \leq \alpha_{i}, 1 \leq i \leq n, \sum_{i=1}^{n} f_{i j} \leq \beta_{j}, 1 \leq j \leq m .
\end{gathered}
$$

Usually, $a_{i}$ and $b_{j}$ are also regarded as space, and cost is involved with space. $\alpha_{i}$ and $\beta_{j}$ are also defined as the weight of some point in the space. EMD has been proved to be a robust distance metric for image retrieval in 2000 [11].

\section{BEDShEET SYSTEM}

In this section, we describe the bedsheet system [1] in this study. The prototype of our bedsheet system is shown in Fig. 1. The system consists of three components: a $64 \times 128$ pressure sensor array, a data sampling unit, and a tablet for data analysis and storage. The sensor array has three layers. The first layer is normal fabric uniformly coated with 64 parallel conductive lines. The middle layer is the eTextile material [12]. The bottom is with 128 parallel conductive lines (perpendicular to the top 64 lines). Thus there are totally 8192 pressure sensors. The data sampling unit scans all the vertical and horizontal lines to produce a resistance map of the entire bedsheet. The tablet converts the resistance map to 8-bits digital values to create a pressure image for further analysis.

\section{APPROACH}

The sleep posture recognition with BEMD is shown in Fig. 2. Pre-processing, BEMD and classification with $k \mathrm{NN}$ are discussed in detail in this section.

\section{A. Pre-processing}

Pre-processing of the pressure image is to remove the difference of position and orientation in the bedsheet as well as height and size of each object. In pre-processing, four steps are adopted. In the first step, the pressure image is filtered with a threshold. Suppose the pressure of each point in the pressure image is $p_{i, j}, 1 \leq i \leq 128,1 \leq j \leq 64$. The threshold is set to $\frac{\sum_{i=1}^{n} \sum_{j=1}^{m} p_{i j}}{N}$, where $N$ is the number of non-zero pressure points. The pressure points with a pressure value smaller than the threshold are assigned to zero. In the second step, a lowpass Gaussian filter of a symmetric $5 \times 5$ unit is applied to remove noises. The third step adopts principal component analysis (PCA) to rotate the pressure image. The rotation angel is equal to the angle between the first eigenvector of the PCA result and the long edge of the pressure image. In the last step, the pressure value of each pressure point is divided by the sum of all pressure points. This is to remove weight difference. Position and height normalization are involved with BEMD, which are discussed in the next subsection.

\section{B. Body-Earth Mover's Distance}

1) Projection: BEMD firstly projects the pressure points to horizontal and vertical directions, and then the definition of space, cost, and weight in BEMD are introduced naturally.

As shown in Fig. 3, the pressure images of supine and left fetus postures are projected to horizontal and vertical directions. Thus two histograms are created for the two directions. The axis $\mathrm{X}$ is the horizontal/vertical axis of the bedsheet, and the axis $\mathrm{Y}$ is the pressure sum of the pressure points that have the same vertical/horizontal axis on the bedsheet.

Though some information is lost due to projections, the difference of supine and left fetus is still obvious as shown in Fig. 3. For left fetus with horizontal projection, there is only one peak in the histogram. While for supine with horizontal projection, the histogram has two peaks. This is due to the fact that the left and right shoulder both bear a large fraction of the total pressure in supine postures. For left fetus with vertical projection, the main two pressure peaks corresponding to the shoulder and the hip have almost the equal total pressure. However for supine with vertical projection, the pressure sum of the two main pressure peaks have a big difference. This 


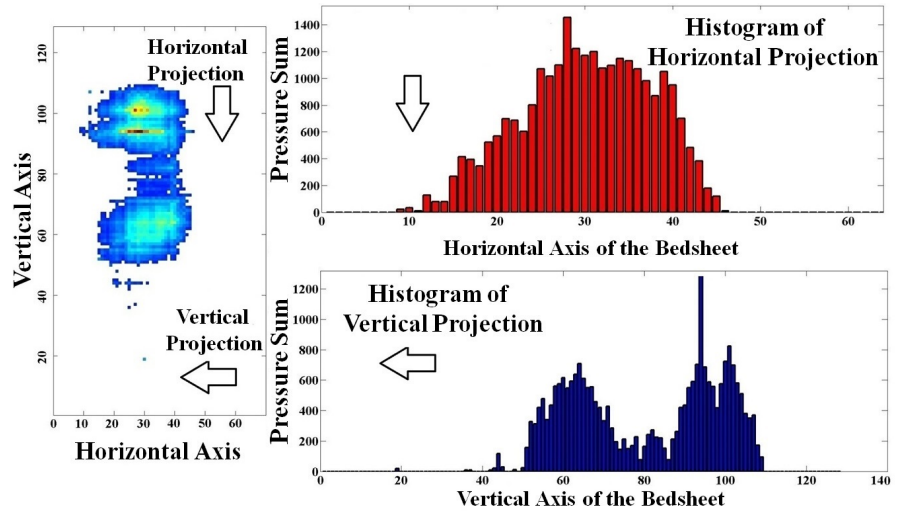

(a)
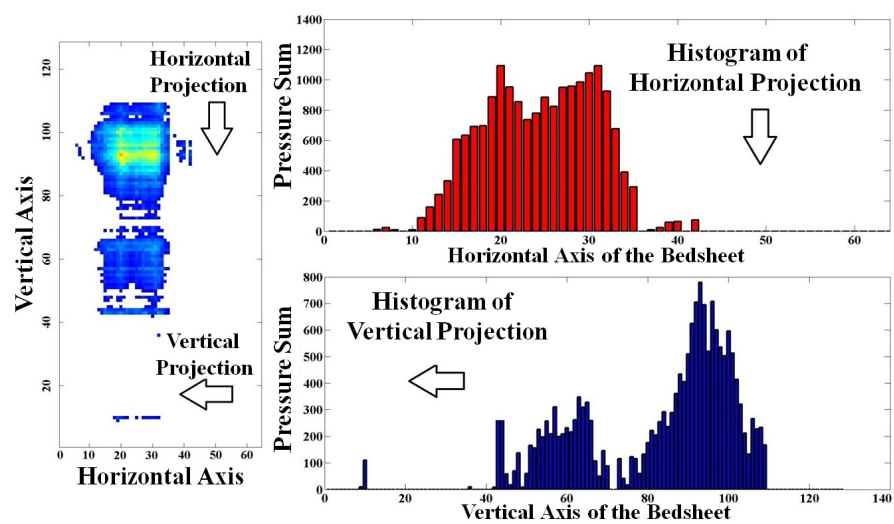

(b)

Fig. 3. Horizontal and vertical projection histograms of (a) left fetus and (b) supine.

may be caused by the fact that shoulder bears more pressure in supine then in left fetus. The analysis of projection histograms of other sleep postures are ignored here due to the limit length of the paper. However, it is obvious that projection histograms are effective to identify the difference of different postures.

With the projection histograms, the space, cost, and weight in BEMD can be easily defined. The space is the horizontal/vertical axis of the bedsheet. As the distance between adjacent parallel conductive lines for horizontal and vertical axis of the bedsheet are the same, the space is a one-dimensional Euclidean space. There are many distance metric for defining the cost in Euclidean space. Squared Euclidean distance, absolute distance, etc., can be chosen. In this approach, absolute distance is selected, which has been proved to be effective in image retrieval [11]. The weight is the pressure. With weight normalization in pre-processing, the weights of different subjects are equal.

2) Region Segmentation: In order to better extra information from pressure images, region segmentation is applied. As shown in Fig. 4, different region segmentation strategies with median point as the segmenting point are presented. In each segmented subregion, the same 2-region or 4-region segmentations can be applied iteratively. How many regions should be segmented is up to specific applications and configurations. In this approach, 4-region segmentation is adopted. In 4-region segmentation, the interference between the upper and lower part of the body on the vertical projection is removed by upperlower segmentation. Furthermore, the dissimilarity introduced by left-right asymmetry of sleep postures can be better measured with left-right segmentation in 4-region segmentation.

3) Translation and Length Normalization: When eight histograms in 4-region segmentation are produced, histogram translation is applied to remove the offset of subjects' locations. For vertical/horizontal projection histograms, the values in $x$ axis is updated minus the vertical/horizontal value of the median point. Length normalization is to remove the difference of height and width of subjects. An assumption is made here that the height of human body is proportional to the width. Thus the scaling ratios in vertical (height) and horizontal (width) projection are equal to the square-root of the ratio of the body areas in the pressure images. The body area is defined as the number of non-zero points in the pressure image.



(a)

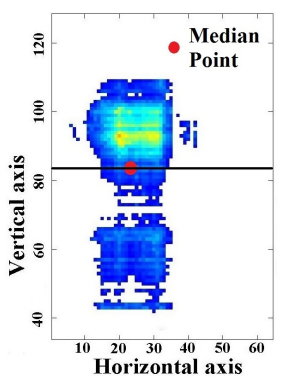

(b)

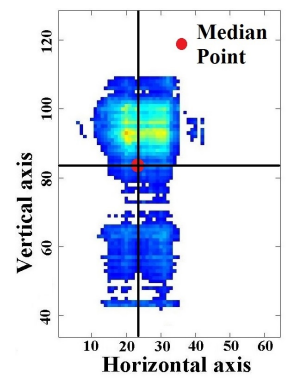

(c)
Fig. 4. Different strategies of region segmentations with median point as the segmenting point: (a) left-right 2-region segmentation, (b) upper-lower 2-region segmentation, and (c) 4-region segmentation.

\section{Classification with $k N N$}

The classification step is divided into two phases. Suppose the training template contains sleep postures of $n$ subjects. The first phase is to apply $1 \mathrm{NN}$ for each person in the training templates. Then each person has its vote for which posture the test data should be. With all the votes, a simple classifier is created. The test posture is assigned with the posture with votes that is more than half of the number of persons in the training templates. Otherwise a skewing rate, $s_{i}, 1 \leq i \leq 6$, is assigned to each posture, which is discussed in detail in Section V-A. Suppose the votes for six sleep postures are $v_{i}, 1 \leq i \leq 6$. The median skewing rate is calculated as $\frac{\sum_{i=1}^{n} s_{i} v_{i}}{6}$. The test posture is assigned to the posture whose skewing rate is nearest to the median skewing rate.

\section{EXPERIMENTS}

\section{A. Experiment setup}

There were 14 subjects in the experiment, where nine were male and five were female. The weight of the subjects ranged from 55 to 85 kilograms, and the height was between 155 and 185 centimeters. The bedsheet system was deployed on a standard twin-size coil spring mattress during the experiment. As shown in Fig. 5, six postures including Left-Log (LL), Left-Fetus (LF), Right-Log (RL), Right-Fetus (RF), Prone (P) and Supine (S) were investigated, which is the same with the previous study [1]. In the data collection, 22 samples were 
recorded for each of the six postures for each subject. Thus there are 132 samples for each subject. Variations in body, arm and leg positions were allowed, and the system was tested on a range of positions that fall within the six postures.

Leave One Out Cross Validation (LOOCV) is used to test the proposed approach. In the experiment, one person's data is selected as the test data, and the rest is regarded as the training data. This process is repeated for each person. The skewing rates of the six postures, LL, LF, RL, RF, $\mathrm{P}$ and $\mathrm{S}$ are $-1,-2$, $+1,+2,0,0$, respectively.

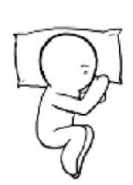

(a)

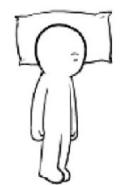

(b)

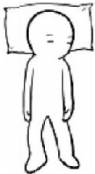

(c)
Right Fetus Right Log Supine

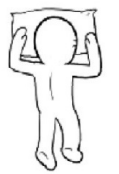

(d)

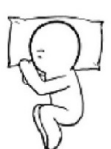

(e)



(f)
Prone Left Fetus Left Log

Fig. 5. Six sleep postures considered in the experiment.

\section{B. Results and Analysis}

The precision and recall results of the experiment are shown in Table I. The average precision of classifications with BEMD is $90.61 \%$, and the average recall is $90.93 \%$. Thus the $f$-measure accuracy is $90.78 \%$. When considering precision and recall, the accuracy of log postures is relatively lower than the other postures. As there is a great variance for arm and leg in log postures, their projection histograms for BEMD have a high possibility to be different from each other, but to be similar with other postures. For supine and prone postures, they have a symmetric pressure distribution in horizontal projection histograms, and their pressure distributions in the vertical projection histograms are different from each other. Thus their accuracies are relatively high. For fetus postures, they have a very asymmetric pressure distribution in horizontal projection histograms (the pressure is mainly distributed on the left/right part of the body for the right/left fetus), then they can be recognized with high accuracies.

A comparison with the previous work is shown in Table II. The proposed BEMD has an accuracy improvement of $9.1 \%$ compared with [1]. The main advantage of BEMD is that it is applied to the raw pressure image data without any feature extractions.

TABLE I. POSTURE CLASSIFICATION CONFUSION MARTRIX $(\%)$

\begin{tabular}{lccccccc}
\hline & LL & LF & P & RT & RF & S & Recall \\
\hline LL & 84.9 & 2.9 & 0.3 & 5.4 & 0 & 6.5 & $\mathbf{8 4 . 8 9}$ \\
LF & 7.6 & 92.4 & 0 & 0 & 0 & 0 & 92.41 \\
P & 1.2 & 1.2 & 94.5 & 0.8 & 0 & 2.3 & 94.55 \\
RL & 1.5 & 0 & 0 & 94.1 & 0.9 & 3.5 & 94.13 \\
RF & 0 & 1.1 & 4.2 & 4.8 & 88.8 & 1.1 & 88.77 \\
S & 3.1 & 0 & 0.4 & 7.6 & 0 & 88.9 & 88.93 \\
\hline Precision & 86.47 & 94.76 & 94.96 & $\mathbf{8 3 . 4 9}$ & 99.02 & 86.91 & $\mathbf{9 0 . 7 8}$ \\
\hline
\end{tabular}

TABLE II. COMPARISON WITH THE PREVIOUS WORK

\begin{tabular}{ccccc}
\hline Approach & \# of Features & \# of postures & Accuracy & Improvement \\
\hline$[1]$ & 32 & 6 & $\mathbf{8 3 . 2} \%$ & N/A \\
This work & N/A & 6 & $\mathbf{9 0 . 7 8} \%$ & $\mathbf{9 . 1} \%$ \\
\hline
\end{tabular}

\section{CONCLUSION}

In this paper, we propose a novel Body-Earth Mover's Distance (BEMD) for sleep posture recognition with a pressure sensitive bedsheet. After pre-processing, the pressure image is segmented into four regions with the median point. With horizontal and vertical projections on each region, eight histograms are created for BEMD, and the similarity between pressure images can be easily obtained. Compared with existing work, no feature extraction is involved. Comprehensive experiments are conducted, and the achieved accuracy of $90.78 \%$ outperforms the previous work [1] with an improvement of $9.1 \%$. In the future work, we will apply the approach into clinical sleep applications, such as sleep stage recognition [13].

\section{ACKNOWLEDGE}

This work is in partially supported by NSF CNS-1423061, ECCS-1462498 and CNS-1547167.

\section{REFERENCES}

[1] J. J. Liu, W. Xu, M.-C. Huang, N. Alshurafa, M. Sarrafzadeh, N. Raut, and B. Yadegar, "A dense pressure sensitive bedsheet design for unobtrusive sleep posture monitoring," in IEEE International Conference on Pervasive Computing and Communications (PerCom'13), San Diego, USA, March 2013, pp. $207-215$.

[2] K. Spiegel, K. Knutson, R. Leproult, E. Tasali, and E. Van Cauter, "Sleep loss: a novel risk factor for insulin resistance and type 2 diabetes," Journal of applied physiology, vol. 99, no. 5, pp. 2008-2019, 2005.

[3] M. E. Thase, "Depression and sleep: pathophysiology and treatment," Dialogues in clinical neuroscience, vol. 8, no. 2, p. 217, 2006.

[4] C. Ambrogio, X. Lowman, M. Kuo, J. Malo, A. R. Prasad, and S. Parthasarathy, "Sleep and non-invasive ventilation in patients with chronic respiratory insufficiency," Intensive care medicine, vol. 35, no. 2, pp. 306-313, 2009.

[5] L. Soban, S. Hempel, B. Ewing, J. N. Miles, and L. V. Rubenstein, "Preventing pressure ulcers in hospitals," Joint Commission Journal on Quality and Patient Safety, vol. 37, no. 6, 2011.

[6] K. Nakajima, Y. Matsumoto, and T. Tamura, "A monitor for posture changes and respiration in bed using real time image sequence analysis," in Engineering in Medicine and Biology Society, 2000. Proceedings of the 22nd Annual International Conference of the IEEE, vol. 1. IEEE, 2000, pp. 51-54.

[7] W.-H. Liao and C.-M. Yang, "Video-based activity and movement pattern analysis in overnight sleep studies," in Pattern Recognition, 2008. ICPR 2008. 19th International Conference on. IEEE, 2008, pp. $1-4$.

[8] A. Sadeh and C. Acebo, "The role of actigraphy in sleep medicine," Sleep medicine reviews, vol. 6, no. 2, pp. 113-124, 2002.

[9] J. J. Liu, W. Xu, M.-C. Huang, N. Alshurafa, M. Sarrafzadeh, N. Raut, and B. Yadegar, "Sleep posture analysis using a dense pressure sensitive bedsheet," Pervasive and Mobile Computing, vol. 10, pp. 34-50, 2014.

[10] S. Ostadabbas, M. Baran Pouyan, M. Nourani, and N. Kehtarnavaz, "In-bed posture classification and limb identification," in Biomedical Circuits and Systems Conference (BioCAS), 2014 IEEE. IEEE, 2014, pp. 133-136.

[11] Y. Rubner, C. Tomasi, and L. J. Guibas, "The earth mover's distance as a metric for image retrieval," International journal of computer vision, vol. 40, no. 2, pp. 99-121, 2000.

[12] W. Xu, M.-C. Huang, N. Amini, L. He, , and M. Sarrafzadeh, "ecushion: A textile pressure sensor array design and calibration for sitting posture analysis," IEEE Sensors Journal, vol. 13, no. 10, pp. 3926-3934, October 2013.

[13] L. Samy, M.-C. Huang, J. J. Liu, W. Xu, and M. Sarrafzadeh, "Unobtrusive sleep stage identification using a pressure-sensitive bed sheet," IEEE Sensors Journal, vol. 14, no. 7, pp. 2092-2101, July 2014. 